\title{
Deubiquitinases in cancer
}

\author{
Rongbin Wei $\mathbf{i}^{1, *}$, Xiaodong Liu ${ }^{2, *}$, Weixin $\mathbf{Y u}^{3, *}$, Tianshu Yang ${ }^{1}$, Wenping Cai ${ }^{1}$, \\ Junjun Liu ${ }^{1}$, Xiao Huang ${ }^{1}$, Guo-tong $X_{u^{1}}{ }^{1}$, Shouliang Zhao ${ }^{3}$, Jianhua Yang ${ }^{1}$, \\ Shangfeng Liu ${ }^{3}$ \\ ${ }^{1}$ Department of Ophthalmology, Shanghai Tenth People's Hospital, Tongji University School of Medicine, Shanghai 200072, \\ P. R. China \\ ${ }^{2}$ Department of Neurosurgery, Huashan Hospital, Fudan University, Shanghai 200040, P. R. China \\ ${ }^{3}$ Department of Stomatology, Huashan Hospital, Fudan University, Shanghai 200040, P. R. China \\ *These authors have contributed equally to this work
}

Correspondence to:

Shangfeng Liu, e-mail: liusf23@gmail.com

Jianhua Yang, e-mail: jianhuay@gmail.com

Shouliang Zhao, e-mail: SLzhao@tongji.edu.cn

Keywords: deubiquitinases, cancer, ubiquitylation, inhibitor

Received: January 16, 2015

Accepted: April 24, 2015

Published: May 07, 2015

\section{ABSTRACT \\ Deubiquitinases are deubiquitinating enzymes (DUBs), which remove ubiquitin from proteins, thus regulating their proteasomal degradation, localization and activity. Here, we discuss DUBs as anti-cancer drug targets.}

\section{INTRODUCTION}

Posttranslational modification of proteins by ubiquitin is a key regulatory event, and the enzymes catalyzing these modifications have been the focus of many studies. Deubiquitinating enzymes (DUBs), which mediate the removal and processing of ubiquitin, might be functionally important but are less well understood. Approximately 100 human DUBs have been identified, over $90 \%$ of which are cysteine-proteases whose catalytic sites contain conserved cysteine $(\mathrm{C})$, histidine $(\mathrm{H})$ and aspartate (D) residues [1]. By regulating the ubiquitin system, DUBs play important roles in multiple biological processes including the cell-cycle, DNA repair, chromatin remodeling, and a wide range of signaling pathways.

Ubiquitylation is reversible process by which ubiquitins are attached to proteins, either singly or in chains. The ubiquitination pathway includes ubiquitinactivating (E1s), ubiquitin-conjugating (E2s) and ubiquitin ligase enzymes (E3s), ultimately responsible for the conjugation of ubiquitin to protein substrates (for reviews see [2,3]). Ubiquitin can be attached to substrate proteins as a single moiety or in the form of polymeric chains in which successive ubiquitin molecules are connected through specific isopeptide bonds. These bonds can be formed on any of the eight primary amines of the ubiquitin molecule (linear/amino $(\mathrm{N})$ terminus/M1, K6, K11, K27, K29, K33, K48 and K63) and thus can achieve a remarkable complexity, termed the ubiquitin code, in which the different chain topologies have distinct signaling functions [4]. The removal of ubiquitins or polyubiquitin chains from the target protein is catalyzed by deubiquitinating enzymes (DUBs). Therefore, DUBs reverse the function of E3 ubiquitin ligases [5]. The human genome encodes approximately 100 DUBs that are subdivided into 5 families based on sequence and structural similarity: ubiquitin-specific proteases (USPs), ubiquitin carboxy-terminal hydrolases (UCHs), ovarian-tumor proteases (OTUs), JAMM Motif Proteases (JAMMs), Machado-Joseph disease protein proteases (MJD) [5].

Ubiquitin (Ub) is a small, highly conserved protein that is added onto primary amino groups of the acceptor protein through an enzymatic cascade involving sequential actions of E1, E2, and E3 conjugating proteins. It is the E3s that are primarily involved in substrate recognition, and, accordingly, there are more E3s than their E1 and E2 counterparts. The E3s can be subdivided into the really interesting new gene (RING) family ( $\sim 300$ in the human genome) and the homologous E6-associated protein carboxy terminus (HECT) family (28 in the human genome) [6]. Both types of E3 link E2 enzymes with substrates, but they differ in that RINGs do not themselves directly transfer ubiquitin, whereas the HECT family forms an intermediate thioester linkage with the ubiquitin $\mathrm{C}$ terminus. Although the RING-E2 interaction occurs at some distance from the E2 catalytic site, it is proposed to facilitate ubiquitin transfer from the E2 by an allosteric mechanism $[7,8]$. 
Deubiquitylation and ubiquitylationare dynamic and reversible post-translational modifications that are involved in the regulation of various cellular pathways. For a long time, functional studies of ubiquitylation have focused on the function of ubiquitylating enzymes, especially the E3 ligases, rather than deubiquitylating enzymes (DUBs) that hydrolyze ubiquitinchains. One reason may be the smaller number of DUBs in the human genome compared to the larger number of E3 ligases, implying broader substrate specificities of DUBs and difficulties identifying the indirect targets. However, recent studies have revealed that DUBs also actively participate in controlling cellular events in cancer. DUBs are also essential for processing ubiquitin precursors and are important for recycling ubiquitin molecules from target protein prior to their degradation and maintaining the free ubiquitin pool in the cell. Here, we will discuss the five different DUB families (USP, UCH, JAMM, OTU, and MJD) and their known biochemical and physiological roles in cancer. In this review, we examine these recent insights, and attempt to provide a comprehensive overview of what is known about this emerging post-translational modification in cancer. In addition, we will propose some directions for future studies.

\section{UBIQUITIN PROTEASOME SYSTEM-TARGETED ANTICANCER THERAPEUTICS}

Covalent attachment of ubiquitin, a highly conserved protein, to a target protein is a means of regulating protein half-life, localization, and activity. Because protein homeostasis is essential for the survival of all cells, but more essential to cancer cells, modulation of individual ubiquitin-proteasome system (UPS) components might present an opportunity for therapeutic targeting. Consequently, many compounds with proteasome inhibitory activity have been developed, including bortezomib (Velcade; Millenium Pharmaceuticals), which is a synthetic dipeptide boronic acid that reversibly inhibits the chymotrypsin-like activity of the $20 \mathrm{~S}$ enzymatic core of the proteasome and induces apoptosis in several malignancies. Velcade is approved by the U.S. Food and Drug Administration (FDA) for the treatment of patients with mantle cell lymphoma and multiple myeloma, even those resistant to doxorubicin, mitoxantrone, melphalan, and dexamethasone, and is commonly used in combination with many of these agents [9]. Amplified protein synthesis (immunoglobulin) in many myeloma cells might underlie their clinical sensitivity to bortezomib and other proteasome inhibitors, because solid tumors do not have a similar commitment to elevated protein synthesis and are not clinically responsive to these drugs. This narrow therapeutic application, combined with some toxicity (sensory neuropathy), might be circumvented by novel proteasome inhibitory molecules [9].

\section{DEUBIQUITINASES AS EMERGING TARGETS FOR ANTICANCER THERAPEUTICS}

Targeted the inhibition of ubiquitin-conjugating enzymes and ligases could offer another therapeutic modality. In addition, screening analyse of ubiquitin ligases reveals G2E3 as a potential target for chemosensitizing cancer cells [10]. Inhibition of NEDD8-related E1 enzyme by MLN4924, E2 enzyme hCdc34 by CC0651, and E3 ligase MDM2 by RITA (NSC652287) and MI-219 reflects this ongoing effort [11]. Deubiquitinases (DUBs) are another class of emerging anticancer target that regulate specific substrate proteins by reversing their ubiquitination through the hydrolysis of isopeptide or a-peptide bonds linking ubiquitin to the target protein [12]. If the target protein is an oncogene, the associated DUB might stabilize its cellular expression, which supports the identification of DUB inhibitors that could reestablish normal protein turnover, location, or activity [12]. Auranofin (Aur) inhibits proteasome-associated deubiquitinases (DUBs) UCHL5 and USP14 rather than the 20S proteasome; inhibition of the proteasome-associated DUBs is required for Aur-induced cytotoxicity; and Aur selectively inhibits tumor growth in vivo and induces cytotoxicity in cancer cells from patients with acute myeloid leukemia [13]. This approach might also avoid the deleterious side effects associated with direct targeting of the proteasome. Genetic and/or functional analysis have revealed DUBs in the category of cancer-associated proteases, and their unique biochemical structures have made them desirable targets for anticancer therapies. In recent studies, more than 40 DUBs have been involved in cancer directly or indirectly. These numbers are not surprising, considering the various critical cellular functions regulated by different DUBs and the diversity of substrates used and regulated by them. A comprehensive list of DUBs altered in different cancers is provided in Table 1.

\section{DEUBIQUITINASES IN CANCER}

It is now well known that DUBs have significant impacts on the regulation of multiple biological processes such as cell-cycle control, DNA repair, chromatin remodeling and several signaling pathways that are frequently altered in cancer $[5,14]$ (Table 1). As a result, different DUB functions are directly and indirectly involved in tumorigenesis.

\section{DUB-associated mutations in cancer}

Recurrent mutations of DUBs are rare in cancer with the exception of CYLD. Germline mutations of the tumor-suppressor gene CYLD are prevalent in familial cylindromatosis, a genetic condition that leads to a predisposition for developing multiple skin tumors [15]. A well-known chromosomal translocation involving 


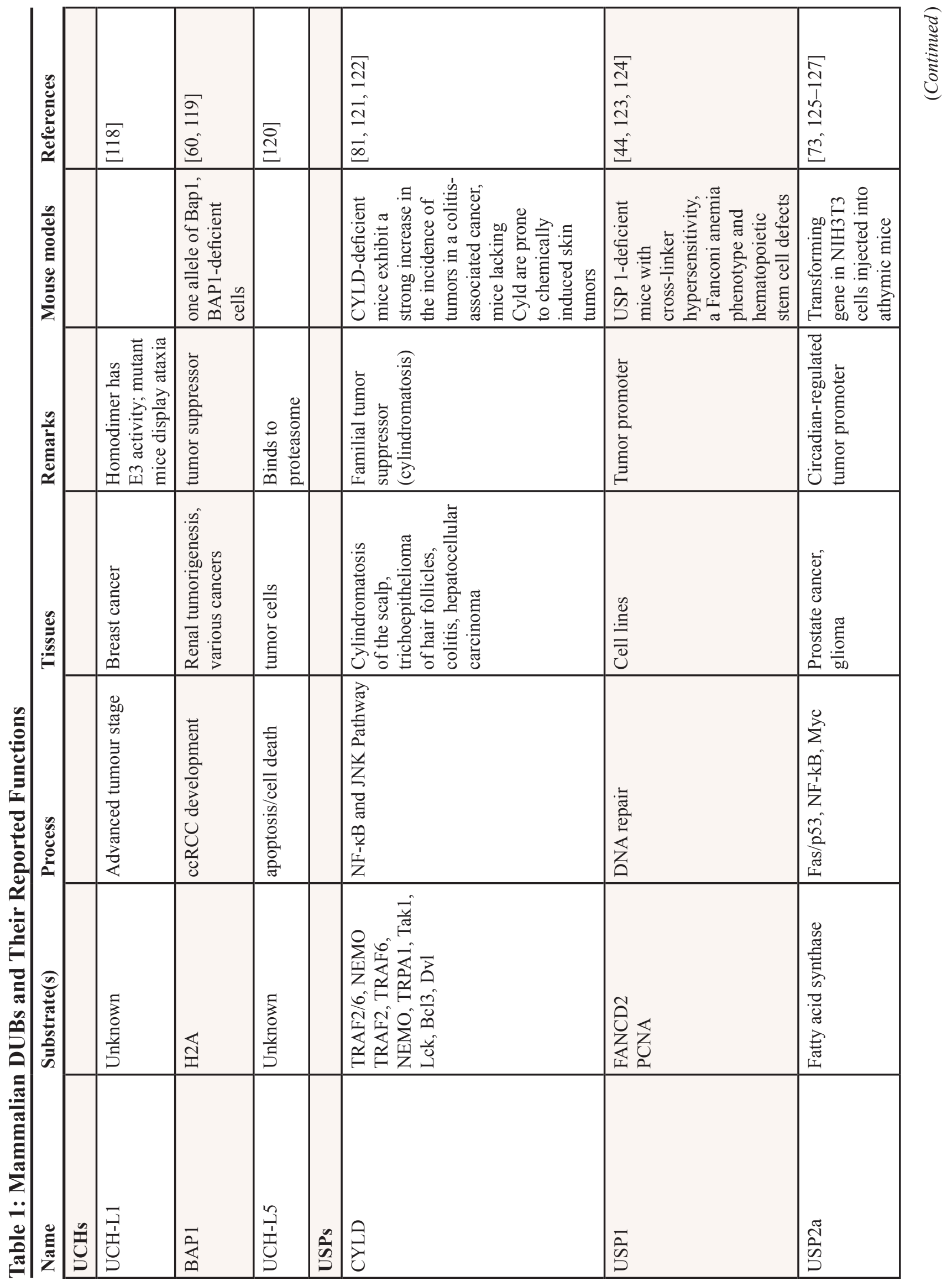




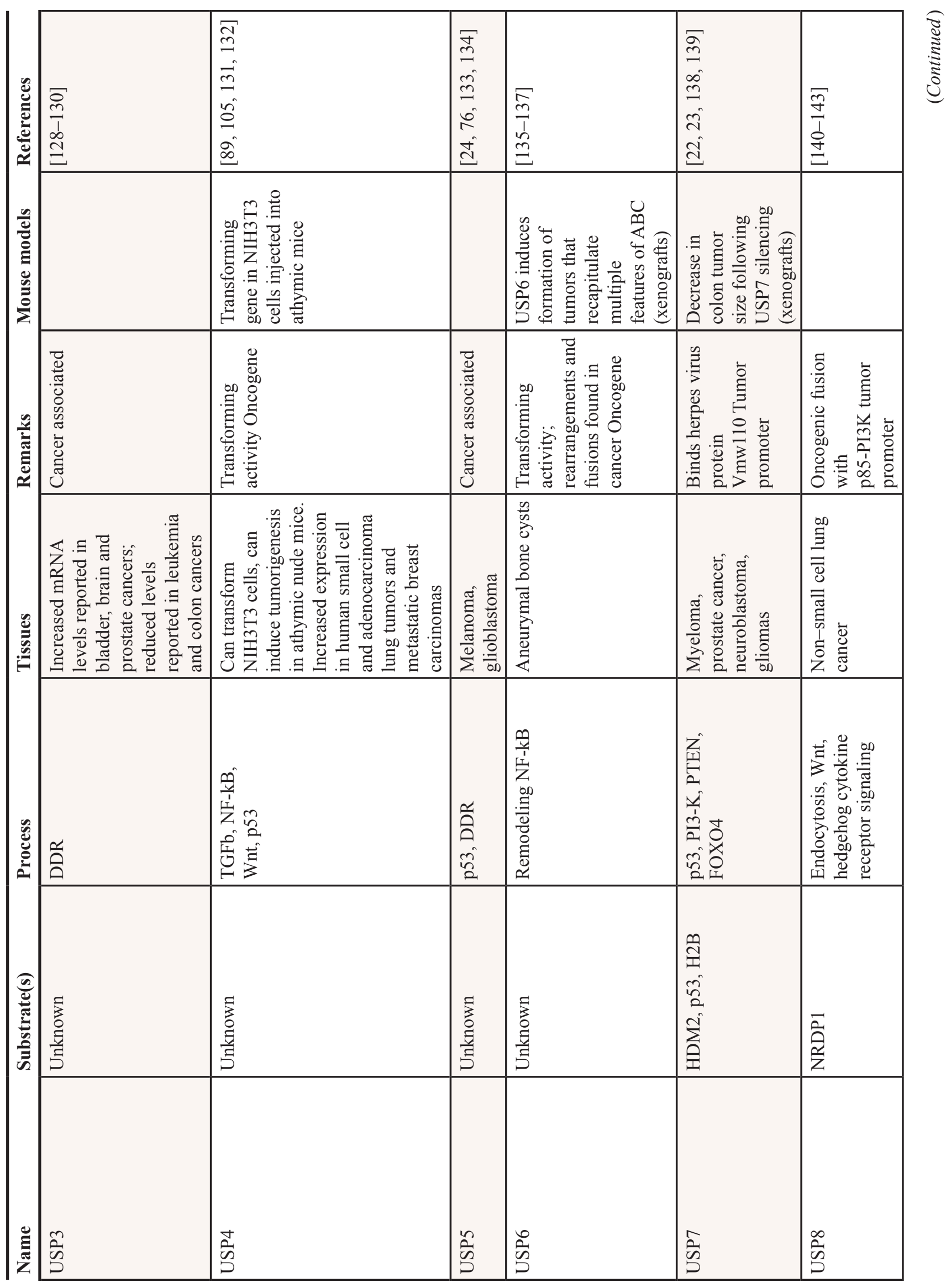




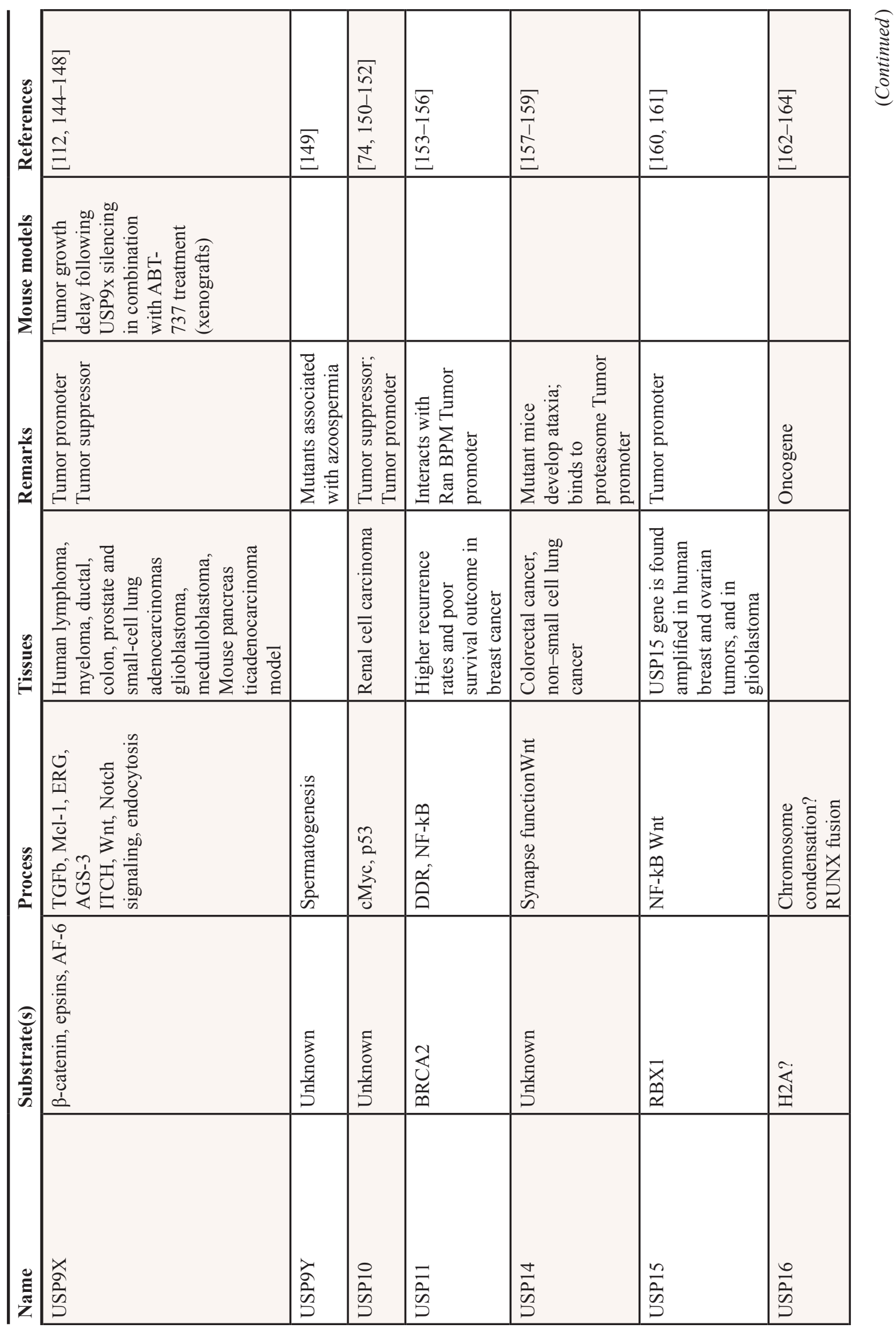




\begin{tabular}{|c|c|c|c|c|c|c|}
\hline 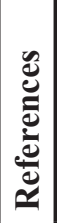 & 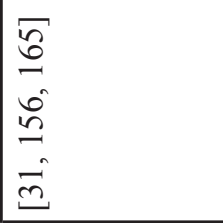 & 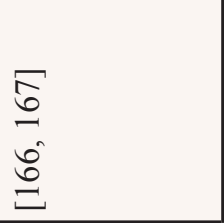 & $\begin{array}{l}a \\
\text { b } \\
\infty \\
0 \\
0\end{array}$ & $\begin{array}{l}\vec{\Xi} \\
\stackrel{2}{\Xi} \\
\Xi\end{array}$ & $\stackrel{\mathbb{N}}{\Xi}$ & $\begin{array}{l}\stackrel{\infty}{\Xi} \\
\stackrel{n}{\unrhd} \\
\Xi\end{array}$ \\
\hline 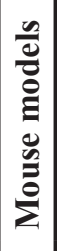 & 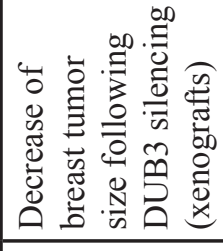 & & & & & \\
\hline 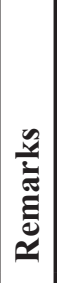 & 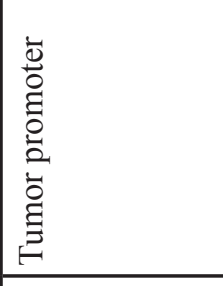 & 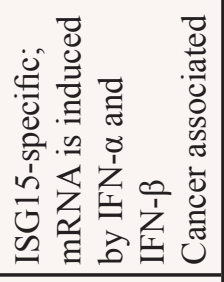 & 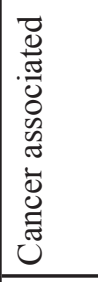 & 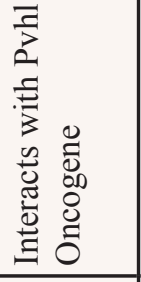 & 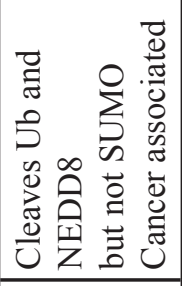 & 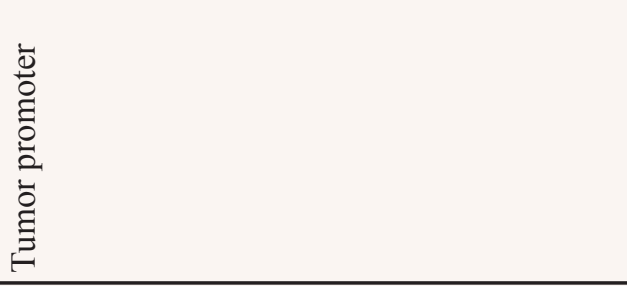 \\
\hline 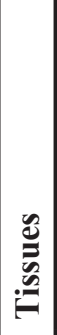 & 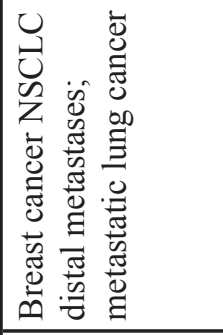 & $\sum_{i}$ & 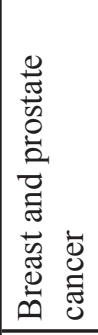 & 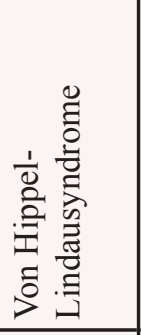 & 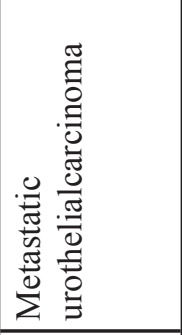 & 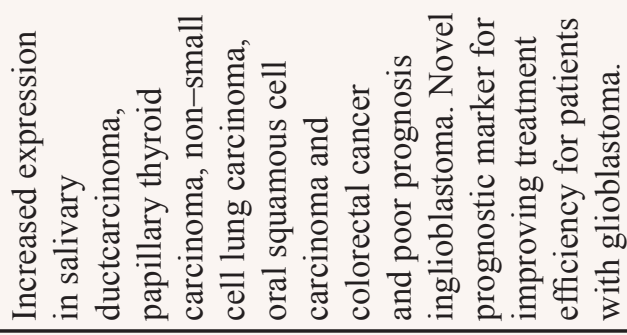 \\
\hline $\mid$ & 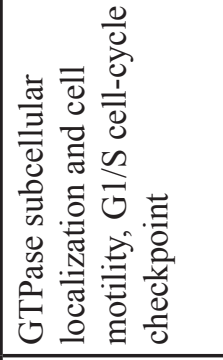 & 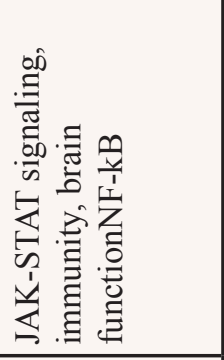 & 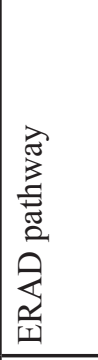 & 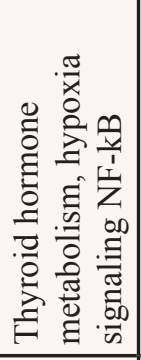 & $\frac{\frac{n}{4}}{\frac{1}{Z}}$ & $\sum_{U^{\prime}}^{\infty}$ \\
\hline 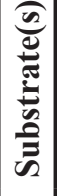 & 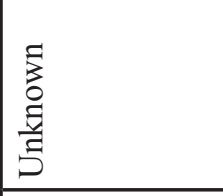 & 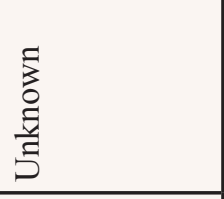 & $\begin{array}{l}\text { F } \\
\text { o } \\
\text { 竘 } \\
5 \\
\end{array}$ & તิ่ & 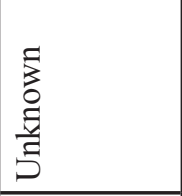 & 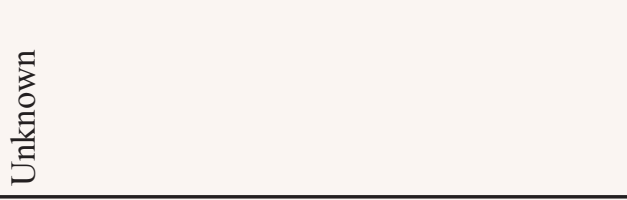 \\
\hline 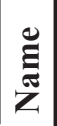 & $\frac{\vec{z}}{\tilde{D}}$ & $\frac{\infty}{a}$ & $\frac{a}{a}$ & 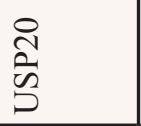 & $\begin{array}{l}\overrightarrow{\widetilde{N}} \\
\tilde{\sim} \\
\vec{D}\end{array}$ & 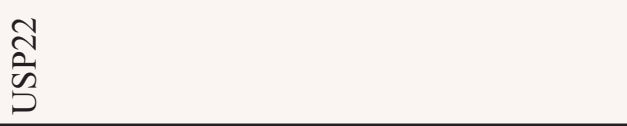 \\
\hline
\end{tabular}




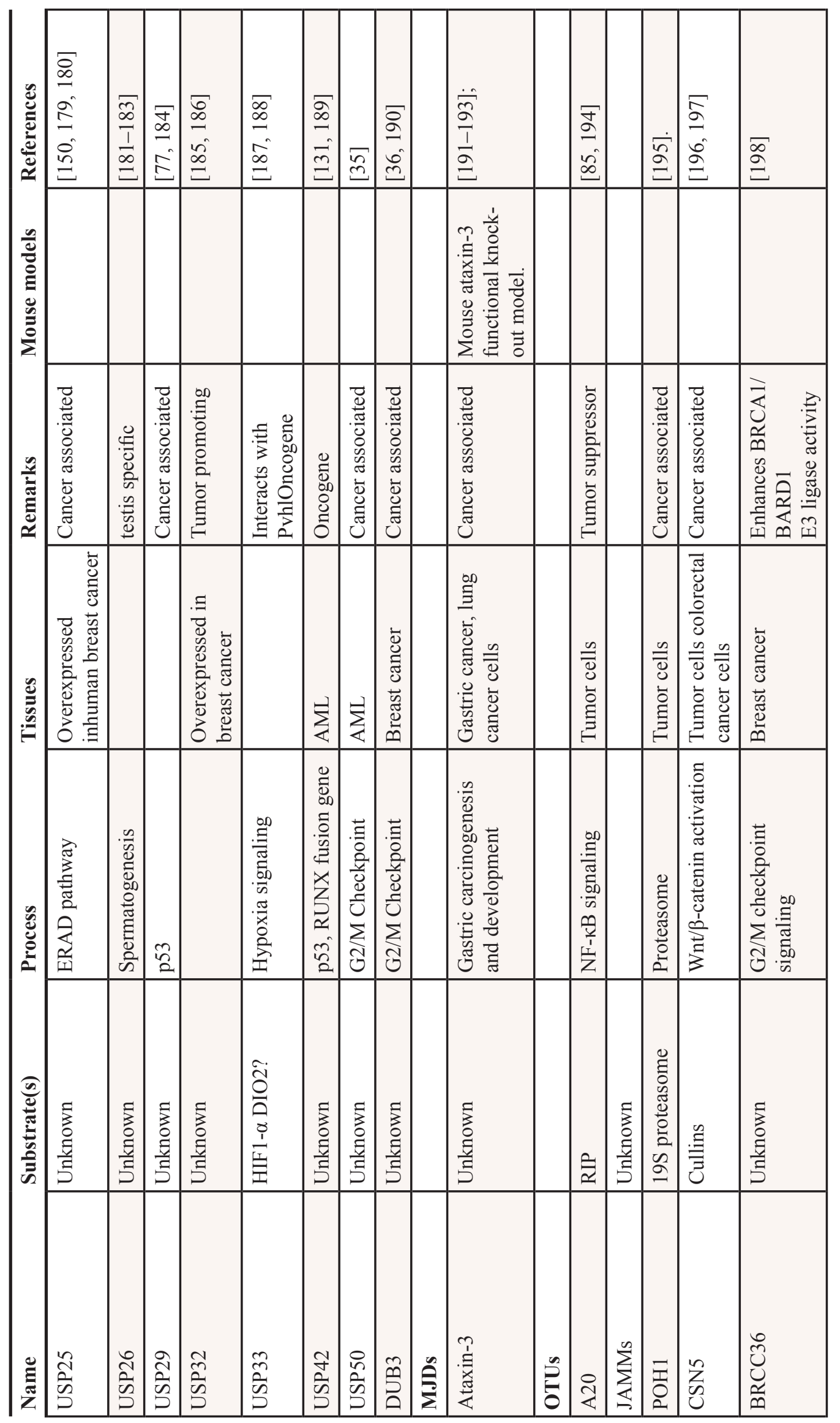


a USP is the fusion of the promoter of CDH11 to the full-length USP6 gene leading to upregulated transcript levels of USP6 [16]. The USP1 amino acid motif $420-520$ is necessary and sufficient for UAF1 binding. USP1 autocleavage can occur in cis, and can be altered by a cancer-associated mutation [17].

\section{Development of small molecule inhibitors against DUBs}

DUBs, as therapeutic targets in cancer and other diseases [14, 18, 19], and DUBs play important roles in recycling ubiquitin monomers to prevent proteasomal degradation of proteins tagged with ubiquitin, and in trimming ubiquitin from tagged proteins [20, 21]. DUBs have been described as a class of anticancer targets; inhibitors of these enzymes were discussed above in the Proteasome section. It is the second function, sparing of target proteins by the removal of conjugated ubiquitin, that has made DUBs attractive targets for cancer and other diseases [20]. In the past decade, tool compounds and/or preclinical development candidate small molecule DUB inhibitors have been reported; these compounds were identified as inhibitors of several DUBs and have a range of reported selectivities with respect to other DUBs and other cysteine proteases [22-24]. Some examples of these inhibitors are given below; their story is a chronicle of the state of DUB-based anticancer drug development up to the present time (Table 2).

\section{Cell-cycle regulation}

Many facts have revealed that DUBs play a critical role in cell-cycle progression since several DUBs are integral components of the core cell-cycle machinery and cell-cycle checkpoints. Functional studies have demonstrated that USP28 play an important part in regulating the stability of $\mathrm{c}-\mathrm{Myc}$, a central modulator of cell growth, proliferation and apoptosis [25]. Some DUBs such as CYLD, USP5, USP13, USP15, USP17, USP37, USP39 and USP44 are essential regulators of events occurring in mitosis. As a consequence, CYLD is crucial for timely entry into mitosis through the regulation of polo-like kinase $1[26,27]$. USP5 in melanoma suppressed cell growth by reinforcing the S/G2-M checkpoint, enhancing extrinsic caspase activation through modulation of p53 and FAS levels and amplifying the apoptotic activity of kinase inhibitors [28]. Moreover, USP13 is recruited by the ubiquitin-recognition protein Ufd1 to antagonize anaphase-promoting complex (APC/C)-Cdh1mediated ubiquitylation of Skp2, thereby accumulating the cyclin-dependent kinase inhibitor p27 and causing a concomitant cell-cycle delay [29]. USP15 stabilizes newly synthesized RE1-silencing transcription factor (REST) and rescues its expression at the mitotic exit [30]. Moreover, USP17 was differentially expressed during the cell cycle, and it was discovered that USP17-knockdown caused a G1 cell cycle block and inhibited the proliferation of tumor-derived cell lines by attenuating GTPase signaling, and that USP17 is tightly regulated during cell division and its expression is necessary to coordinate cell cycle progression [30, 31]. Deubiquitinase USP37 is activated by $\mathrm{CDK} 2$ to antagonize $\mathrm{APC}(\mathrm{CDH} 1)$ and promote $\mathrm{S}$ phase entry [32]. USP39 is essential for mitotic spindle checkpoint integrity and depletion of USP39 in mRNA processing contributes to a specific reduction in Aurora B-mRNA levels [33]. USP44 prevents the premature activation of $\mathrm{APC} / \mathrm{C}$ by stabilizing the $\mathrm{APC} / \mathrm{C}$-inhibitory Mad2-Cdc20 complex through deubiquitylation which contributes to the generation of the switch-like transition controlling anaphase entry [34]. In contrast, USP50 is involved in the G2/M checkpoint and serves as a regulator of HSP90-dependent Wee1 stability to suppress entry into mitosis [35]. Furthermore, USP17L2 (known as DUB3)

Table 2: Experimental DUB inhibitors

\begin{tabular}{|l|l|l|}
\hline DUBs & Compound & \multicolumn{1}{l}{ References } \\
\hline USP1 & Pimozide; GW7647; ML323 and ML323 $(70)$ & {$[11,199]$} \\
\hline USP5 & WP1130, EOAI3402143 (G9); Vialinin A & {$[24,28,200]$} \\
\hline USP7 & $\begin{array}{l}\text { HBX41, 108, HBX19,818; P0050429 and } \\
\text { WO2013030218 }\end{array}$ & {$[22,23,201]$} \\
\hline USP8 & HBX 41,108 & {$[202]$} \\
\hline USP9X & WP1130;EOAI3402143 (G9) & {$[24]$} \\
\hline USP11 & Mitoxantrone & {$[24]$} \\
\hline USP14 & WP1130, b-AP15, Auranofin & {$[24,120,203,204]$} \\
\hline UCH37 & WP1130 & {$[24]$} \\
\hline UCH-L1 & UCH-L1inhibitor1 & {$[205]$} \\
\hline UCHL5 & b-AP15, Auranofin & {$[203,204]$} \\
\hline DUB (pan) & 12PGJ2; PR-619 & {$[206,207]$} \\
\hline
\end{tabular}


deubiquitylates and is responsible for the stabilization of Cdc25A to promote oncogenic transformation in human breast cancers. In addition, USP17L2 is an example of a transforming ubiquitin hydrolase that subverts a key component of the cell cycle machinery [36]. Moreover, USP2 directly interacts with cyclin D1 and promotes its stabilization by antagonizing ubiquitin-dependent degradation. In addition, targeting USP2 is an effective approach to induce growth suppression in cancer cells with aberrant overexpression of cyclin D1 [37]. USP19 regulates cell proliferation and p27(Kip1) levels in a cell context-dependent manner through both E3 ligase KPC1dependent and KPC1-independent mechanisms [38]. Likewise, USP7 (a mdm2 regulator of p53 function) is essential in cell proliferation and differentiation, through its regulatory activity on phosphatase and tensin homolog and FOXO localization [39, 40]. Moreover, CSN5/ Jab1 acts as a modulator of the mammalian cell cycle, preventing senescence and endocycle as well as the proper progression of the somatic cell cycle [41]. Finally, BAP1 forms complexes with the transcription factors Yin Yang 1 (YY1) and host cell factor 1 (HCF-1), and controls cellcycle progression at $\mathrm{G} 1 / \mathrm{S}$ by co-regulating transcription from $\mathrm{HCF}-1 / \mathrm{E} 2 \mathrm{~F}$-governed promoters $[42,43]$.

\section{DNA damage repair}

The link between DNA damage repair and tumor development is demonstrated by the increased cancer rates reported for those disorders involving deficient DNA repair mechanisms, such as Fanconi anemia. USP1 is responsible for DNA damage repair by regulating Fanconi anemia protein (FANCD2) through deubiquitylation and the subsequent stabilization of checkpoint kinase 1 (CHK1) [44, 45]. Furthermore, USP1 controls proliferating cell nuclear antigen ubiquitylation, a safeguard factor against error-prone DNA translesion synthesis that is ubiquitylated and responsible for genotoxic stress [46]. As a result, USP1 forms a complex with U2 small nuclear ribonucleoparticle auxilliary factor 1 and promotes doublestrand break repair through homologous recombination [47]. Moreover, DUB UCHL5 regulates DNA doublestrand breaks (DSBs) resection and repair by homologous recombination through protecting its interactor, NFRKB, from degradation [48]. Dub3 controls DNA damage signaling by directly deubiquitinating $\mathrm{H} 2 \mathrm{AX}$ [30]. Other DUBs are implicated in the regulation of DNA repair. Thus, BRCC36, Dub3, USP3, USP16, USP44 and OTUB1 participate in regulating the RNF8/RNF168 pathway of double strand breaks repair [49], and USP5 links the suppression of p53 and FAS levels in melanoma to the BRAF pathway [50]. USP11 is involved in the cellular response to mitomycin $\mathrm{C}$-induced DNA damage within the BRCA2 pathway signaling [51]. USP20 is a novel regulator of ATR-dependent DNA damage [52]. USP20 serves as a novel regulator of ATR-dependent DNA damage checkpoint signaling through the deubiquitination and stabilization of claspin and enhances the activation of ATR-Chk1 [53]. Moreover, USP28 is required to stabilize Chk2 and 53BP1 in response to DNA damage; both USP 28 and Chk2 are required for DNA-damage-induced apoptosis, and they accomplish this in part through the regulation of the p53 induction of proapoptotic genes such as PUMA [54]. USP34 promotes a feed-forward loop to enforce ubiquitin signaling at DNA doublestrand breaks (DSBs), and highlights the critical roles of ubiquitin dynamics in genome stability maintenance [55]. Finally, USP47 has been identified as the enzyme responsible for the deubiquitylation of the base excision repair DNA polymerase $(\operatorname{Pol} \beta)$, thus playing an important role in regulating DNA repair and maintaining genome integrity [56]. In the last decade, protein alterations, such as deubiquitination, have emerged as key modifications in the control of DNA damage response (DDR) signaling.

\section{Chromatin remodeling}

Some DUBs interact with histones, predominately $\mathrm{H} 2 \mathrm{~A}$ and $\mathrm{H} 2 \mathrm{~B}$, the post-translational modifications of which control chromatin structure dynamics and gene expression, which are processes that are frequently altered in cancer. To the best of our knowledge, there are at least eight DUBs that can deubiquitylate histones including BAP1, USP3, USP7, USP16, USP21, USP22, MYSM1 and BRCC36 [57-60]. Both H2A and H2B are deubiquitylated by these DUBs, although $\mathrm{H} 2 \mathrm{~A}$ is preferentially targeted [61]. MYSM1, USP7, USP22 and BRCC36 are part of the 2A-DUB, polycomb-repressive complex 1, SAGA and BRCA1-A multisubunit complexes, respectively [62-65]. However, studies have not identified USP3 and USP16 as being involved in any of these complexes, suggesting that their chromatin-regulatory mechanisms might be different. Apart from histones, gene transcription can be regulated by the deubiquitylation of other chromatin-associated substrates. Therefore, USP22 regulates the protein stability of telomeric-repeat binding factor 1 [66]. Nevertheless, MEL18 and BMI were deubiquitylated by USP7 and USP11, two chromatinbound components of polycomb-repressive complex 1 complex components that influence the transcriptional regulation of p16INK4a [65]. Furthermore, UCHL5, the activation of which is crucial for the proteasome, also interacts with the human lno80 chromatin-remodeling complex [67]. Finally, as a result, BAP1 deubiquitylates the chromatin-related protein host cell factor 1, which regulates transcription by linking histone-modifying enzymes to a subset of transcription factors [43].

\section{Signaling pathways}

Recent studies have recognized the fact that it is more important to focus on signaling pathway rather than on individual genes altered in cancer [68]. 
Therefore, mutations in individual genes associated with the same cancer-relevant signaling pathway have been demonstrated in many tumors and are known to have similar functional effects, providing a wide range of drug targets [69]. Some signaling pathways are recurrently altered in many cancers, as those involving p53, NF-kB, receptor tyrosine kinases (RTKs), Wnt, transforming growth factor-b (TGF- $\beta$ ) IFN and Akt, which are significantly influenced by the activity of DUBs.

\section{The p53 signaling pathway}

p53 is, a tumor suppressor that is well studied because it has critical functions in maintaining cellular homeostasis and is frequently mutated in most tumors [70]. To date, research studies have reported many DUBs that are associated with p53 regulation: USP2, USP4, USP5, USP7, USP10 and USP29. USP7 is involved in the dynamic regulation of the p53-MDM2 pathway by controlling the stability of both p53 and MDM2, a ubiquitin ligase that also contributes to the maintenance of p53 ubiquitylation levels $[71,72]$. Therefore, USP7 can be regarded as an oncogene or a tumor suppressor depending on whether it mainly deubiquitylates MDM2 or p53, respectively. Similarly, USP2 influences the stabilization of MDM2, but in contrast to USP7, does not deubiquitylate p53 [73]. USP10 is also associated with regulation of p53 localization and stability, however, unlike USP2 and USP7, it does not interact with MDM2 [74]. Otub1, a DUB from the OTU-domain containing protease family abrogates p53 ubiquitination and stabilizes and activates p53 in cells independent of its deubiquitinating enzyme activity [75]. Interestingly, USP10 can stabilize both mutated and wild-type $p 53$, thus having a dual role in tumorigenesis depending on p53 status. Likewise, USP5 has been identified as a potential target for p53 activating therapeutic agents for the treatment of cancer [76]. Moreover, USP29 deubiquitylates and stabilizes p53 in response to oxidative stress [77]. Furthermore, USP4 interacts directly with and deubiquitinates ARF-BP1, leading to the stabilization of ARF-BP1 and subsequent reduction of p53 levels [78]. Taken together with the finding that USP4-deficient murine embryonic fibroblasts (MEFs) exhibit retarded growth, premature senescence, hyperactive DNA damage checkpoints and resistance to oncogenic transformation, thus suggesting that USP4 is a potential oncogene [78].

\section{The nuclear factor-kappa B (NF-kB) signaling pathway}

It is well-known that the nuclear factor-kappa B (NF-kB) signaling pathway has multiple roles in cancer progression including anti-apoptosis, cell cycle, angiogenesis and metastasis [79]. Several DUBs such as A20, CYLD, MCPIP1, USP2, USP4, USP11, USP15,USP21 and USP34 are involved in NF-kB signaling by acting on several components of the pathway [80]. A20 and CYLD regulate levels of ubiquitin related to TRAF6. Furthermore, CYLD is also associated with the deubiquitylation of TGF-B-activated kinase 1 [81], B-cell CLL/lymphoma 3 (Bcl-3) [82] and mitogenactivated protein kinases [83], whereas A20 promotes the degradation of TRAF2 in lysosomes by means of its own E3 ligase activity [84]. A20 also potentiates the proteasomal degradation of RIPK1 through Lys48 polyubiquitylation, whereas its OUT domain removes Lys63-linked ubiquitin chains of RIPK1, leading to the downregulation of NF-kB signaling [85]. The interactions between CYLD and OTU deubiquitinases with linear linkage specificity (OTULIN) and the linear ubiquitin chain assembly complex (LUBAC ) ligase are involved in controlling the extent of TNF-a-induced NF-kB activation in cells by fine-tuning the generation of linear ubiquitin chains by LUBAC [86]. MCPIP1 negatively controls c-Jun N-terminal kinase and NF-kB activity through deubiquitylation of TRAF2, TRAF3 and TRAF6, thus playing an essential role in the regulation of inflammatory signaling [87]. USP2 has been reported as a regulator of TNF $\alpha$-induced NF-kB signaling, and is required for $\mathrm{Ikb}$ phosphorylation, NF-kB nuclear translocation and NF-kB-dependent target genes expression [88]. USP4 also plays an essential role in the downregulation of $\mathrm{TNFa}-$ induced NF-kB activation through deubiquitylation of TGF-b-activated kinase 1 [89]. Furthermore, OTUD5 deubiquitylates TRAF3 resulting in diminished type I interferon and interleukin-10 responses [90]. Moreover, USP15 stabilizes IkBa by inhibiting its degradation by the proteasome [1] and USP11 interacts with IkB kinase a, an inhibitor of NF-kB, upon induction by TNF $\alpha$ [1]. USP21 inhibits NF-kB activation through the deubiquitylation of RIPK1 [91]. Likewise, Cezanne suppresses NF-kB nuclear translocation and transcriptional activity by deubiquitylating RIPK1 signaling intermediaries and interacting with DJ-1 [92, 93]. USP34 silencing spared upstream signaling but led to a more pronounced degradation of the NF- $\kappa \mathrm{B}$ inhibitor $\mathrm{I} \kappa \mathrm{B} \alpha$, and culminated in an increased DNA binding activity of the transcription factor [94].

\section{RTK signaling pathways}

Recent studies have found that the relevance of receptor tyrosine kinase (RTK)-mediated signal transduction pathways (RTKs) in several human tumor types is reflected by multiple abnormalities in RTK-dependent pathways [95]. To date, numerous oncogenic mechanisms are known to interfere with RTK internalization. There are at least five DUBs: USP8, USP17, USP18, AMSH and POH1-that affect the trafficking of RTKs including epidermal growth factor receptor (EGFR), Met and ErbB2. USP8 has an important role in the stabilization of RTKs through 
deubiquitylation, allowing their recycling to the plasma membrane [96]. USP8 depletion might reflect the loss of ESCRT-0 components which associate with the retromer components Vps35 and SNX1, whereas failure to deliver lysosomal enzymes efficiently might also contribute to the observed block in receptor tyrosine kinase degradation [97]. Furthermore, the endosome-associated AMSH (also known as STAMBP) promotes EGFR recycling at the expense of lysosomal sorting [98, 99]. Moreover, USP18 has been identified as a new regulator of EGFR synthesis by controlling its translation [100]. Recent research has shown that USP18 controls EGFR expression and cancer cell survival depending on the transcriptional activation and mRNA stabilization of miR-7 host genes [101]. Finally, another RNA interference screen has identified POH1 as a regulator of ubiquitylated ErbB2 levels, although it is not associated with its turnover [102].

\section{The Wnt signaling pathway}

The Wnt signaling pathway is required for regulation of embryonic development and is frequently activated in cancer [103]. To date, there are at least eight DUBs involved in this pathway, including CYLD, USP4, USP7, USP14, USP15, USP34, TRABID and OTULIN. In fact, CYLD serves as a negative regulator of Wnt signaling and $\beta$-catenin activation by deubiquitylating the cytoplasmic effector Dishevelled (Dvl) [104]. USP4 negatively regulates Wnt signaling by interacting with Nemolike kinase [105] and USP15 promotes $\beta$-catenin degradation through the stabilization of adenomatous polyposis coli (APC), a negative regulator of Wntmediated transcription [106]. USP7 interacts with RNF220 by stabilizing $\beta$-catenin, leading to the RNF220/USP7 complex deubiquitinating $\beta$-catenin and enhancing canonical Wnt signaling [107]. USP14 functions as a positive regulator of the Wnt signaling pathway. Tissue microarray analysis of colon cancer has consistently revealed a strong correlation between the levels of USP14 and $\beta$-catenin, which suggests an oncogenic role for USP14 via the enhancement of Wnt/ $\beta$-catenin signaling [108]. In contrast, USP34 serves as a positive modulator of Wnt signaling by inhibiting $\beta$-catenin-dependent transcription [109]. Furthermore, TRABID is a DUB that is critically involved in T-cell factor (TCF)-mediated transcription of Wnt genes by deubiquitylating APC [110]. The interaction of HOIP with OTULIN is also involved in OTULIN suppressing the canonical Wnt signaling pathway activation by LUBAC [86].

\section{The transforming growth factor- $\beta$ signaling pathway}

Transforming growth factor- $\beta$ (TGF- $\beta$ ) is a multifunctional protein that plays a dual role in oncogenesis by serving as an antiproliferative factor at early stages and promoting epithelial-to-mesenchymal transition at later stages [111]. To date, many DUBs have been reported to be involved in the regulation of the TGF $\beta$ pathway, including USP4, USP11, USP15, USP18, USP9X and CYLD. The UCH member UCH37, OTU members A20 and OUTB1 and JAMM/JPN+ member AMSH, are also reported to regulate the TGF $\beta$ pathway. USP9X positively regulates TGF- $\beta$ signaling by deubiquitylating SMAD4 and promoting its association with SMAD2 [112]. USP9X regulates cell polarity and proliferation and modulates their phosphorylation and activation by LKB1 through deubiquitylation of the AMPK-related kinases NUAK1 and MARK4 [113]. Finally, AMSHLP and UCHL5 promote TGF- $\beta$ responses through their interaction with inhibitory I-SMADs $[113,114]$.

\section{The IFN pathway}

The IFN immune system consists of type I, II, and III IFNs, signals through the JAK-STAT pathway, and plays a central role in host defense against viral infection. Posttranslational modifications like ubiquitination regulate diverse molecules in the IFN pathway. USP13 was the first DUB identified to modulate STAT1 and play a role in the antiviral activity of IFN against DEN-2 replication [115]. USP $2 b$ negatively regulates IFN- $\beta$ signaling and the antiviral immune response by deubiquitinating K63-linked polyubiquitin chains from TBK1 to terminate TBK1 activation [116].

\section{The Akt pathway}

USP12, in complex with Uaf-1 and WDR20, directly deubiquitinates and stabilizes the Akt phosphatases PHLPP and PHLPPL resulting in decreased levels of active pAkt. Depleting USP12 sensitizes prostate cancer cells to therapies aimed at Akt inhibition irrespective of their sensitivity to androgen ablation therapy [117].

\section{DISCUSSION AND FUTURE DIRECTION}

Conclusively, there is accumulating experimental evidence that a large number of DUBs play important role in several stages of cancer development and progression. The potential to influence processes including signal transduction, proliferation and apoptosis by affecting ubiquitination and proteasomal degradation of key regulators is both promising and exciting. DUBs are more likely drug candidates than E3 ligases due to the lack of defined catalytic residues in the latter. Most DUBs are cysteine enzymes, which should be easy to use as target drugs, particularly if using compounds containing Michael acceptors. Furthermore, many DUBs show profound changes in their expression levels in different malignant tumors. As a result, together with the recent success of clinically targeting the ubiquitin proteasome 
system in cancer, DUBs have emerged as appealing targets in the development of novel specific therapies against human malignancies. To date, no DUB-targeted strategies have reached clinical trials and many challenges remain before translating this information into clinical benefits for cancer patients. In addition, DUBs could be targeted for anticancer therapeutics. Early evidence for antitumor efficacy with the currently available DUB inhibitors is more than encouraging and sets the stage for the development of selective, as well as partially selective, small-molecule DUB inhibitors. Moreover, the assignment of oncogenic or tumor-suppressive roles to certain DUBs is still due to the cellular context and further analysis will be required for functional and clinical validation of DUBs as drug targets. Bioavailability is a hurdle that needs to be overcome, but if progress can be made in this area molecules containing functional dienones as well as other types of compounds might be developed into useful cancer therapeutics. Finally, the generation of gain- or loss of- function animal models for selected DUB family members will likely contribute to clarify the relative relevance of individual DUBs and their alterations in tumorigenic progression. Hopefully, our review derived from many studies will provide novel insights into the multiple questions associated with DUBs and lead to the introduction of DUB-targeting therapy in cancer treatments and crucial components of molecular therapies against cancer.

\section{ACKNOWLEDGMENTS}

We thank Shaobo Su (Tongji University School of Medicine) for critical discussion for conception and design. This work was supported by the Ministry of Science and Technology of China (2013CB967500), the National Natural Science Foundation of China (31401089,31170824), the Natural Science Foundation of Shanghai (12ZR1434200).

\section{CONFLICTS OF INTEREST}

The authors declare no conflict of interest.

\section{Author contributions}

R. W. and X. L.: manuscript writing and editing; T. Y., W. Y and X. H.: critical discussion for conception and design; S. L., S. Z and J. Y.: conception and design, manuscript writing and editing, final approval of manuscript.

\section{REFERENCES}

1. Fraile J, et al., Deubiquitinases in cancer: new functions and therapeutic options. Oncogene. 2011; 31:2373-2388.
2. Pickart CM. Mechanisms underlying ubiquitination. Annual review of biochemistry. 2001; 70:503-533.

3. Kerscher O, Felberbaum R, Hochstrasser M. Modification of proteins by ubiquitin and ubiquitin-like proteins. Annu. Rev. Cell Dev. Biol. 2006; 22:159-180.

4. Ikeda F, Dikic I. Atypical ubiquitin chains: new molecular signals. EMBO reports. 2008; 9:536-542.

5. Komander D, Clague MJ, Urbé S. Breaking the chains: structure and function of the deubiquitinases. Nature reviews Molecular cell biology. 2009; 10:550-563.

6. Li W, et al., Genome-wide and functional annotation of human E3 ubiquitin ligases identifies MULAN, a mitochondrial E3 that regulates the organelle's dynamics and signaling. PloS one. 2008; 3:e1487.

7. Budhidarmo R, Nakatani Y, Day CL. RINGs hold the key to ubiquitin transfer. Trends in biochemical sciences. 2012; $37: 58-65$.

8. Özkan E, Yu H, Deisenhofer J. Mechanistic insight into the allosteric activation of a ubiquitin-conjugating enzyme by RING-type ubiquitin ligases. Proceedings of the National Academy of Sciences of the United States of America. 2005; 102:18890-18895.

9. Chen D, et al., Bortezomib as the first proteasome inhibitor anticancer drug: current status and future perspectives. Current cancer drug targets. 2011; 11:239.

10. Schmidt F, et al., Screening analysis of ubiquitin ligases reveals G2E3 as a potential target for chemosensitizing cancer cells. Oncotarget. 2014.

11. Mattern MR, Wu J, Nicholson B. Ubiquitin-based anticancer therapy: carpet bombing with proteasome inhibitors vs surgical strikes with E1, E2, E3, or DUB inhibitors. Biochimica et Biophysica Acta (BBA)Molecular Cell Research. 2012; 1823:2014-2021.

12. Hussain S, Zhang Y, Galardy PJ. DUBs and cancer. Cell Cycle. 2009; 8:1688-1697.

13. Liu N, et al., Clinically used antirheumatic agent auranofin is a proteasomal deubiquitinase inhibitor and inhibits tumor growth. Oncotarget. 2014; 5:5453.

14. Turcu FER, Ventii KH, Wilkinson KD. Regulation and cellular roles of ubiquitin-specific deubiquitinating enzymes. Annual review of biochemistry. 2009; 78:363.

15. Bignell GR, et al., Identification of the familial cylindromatosis tumour-suppressor gene. Nature genetics. 2000; 25:160-165.

16. Oliveira AM, et al., Aneurysmal bone cyst: a neoplasm driven by upregulation of the USP6 oncogene. Journal of Clinical Oncology. 2006; 24:e1-e1.

17. Olazabal-Herrero A, García-Santisteban I, Rodríguez JA. Structure-function analysis of USP1: insights into the role of Ser313 phosphorylation site and the effect of cancerassociated mutations on autocleavage. Molecular cancer. $2015 ; 14: 33$.

18. Shi D, Grossman SR, Ubiquitin becomes ubiquitous in cancer: emerging roles of ubiquitin ligases and 
deubiquitinases in tumorigenesis and as therapeutic targets. Cancer Biol Ther. 2010; 10:737-747.

19. Nicholson B, et al., Deubiquitinating enzymes as novel anticancer targets. 2007.

20. Ciechanover A, Orian A, Schwartz AL. The ubiquitin-mediated proteolytic pathway: Mode of action and clinical implications. Journal of cellular biochemistry. 2000; 77:40-51.

21. Lee MJ, et al., Trimming of ubiquitin chains by proteasomeassociated deubiquitinating enzymes. Molecular \& Cellular Proteomics. 2011; 10:R110. 003871.

22. Colland F, et al., Small-molecule inhibitor of USP7/HAUSP ubiquitin protease stabilizes and activates p53 in cells. Molecular cancer therapeutics. 2009; 8:2286-2295.

23. Reverdy C, et al., Discovery of specific inhibitors of human USP7/HAUSP deubiquitinating enzyme. Chemistry \& biology. 2012; 19:467-477.

24. Kapuria V, et al., Deubiquitinase inhibition by small-molecule WP1130 triggers aggresome formation and tumor cell apoptosis. Cancer research. 2010; 70:9265-9276.

25. Popov N, et al., The ubiquitin-specific protease USP28 is required for MYC stability. Nature cell biology. 2007; 9:765-774.

26. Stegmeier F, et al., The tumor suppressor CYLD regulates entry into mitosis. Proceedings of the National Academy of Sciences. 2007; 104:8869-8874.

27. Jaworski J, et al., USP17 is required for clathrin mediated endocytosis of epidermal growth factor receptor. Oncotarget. 2014; 5:6964.

28. Potu H, et al., Deubiquitinases Usp9x and Usp5 control tumorigenicity and apoptotic responsiveness in malignant melanoma. Cancer Research. 2014; 74:4435-4435.

29. Chen M, Gutierrez GJ, Ze'ev AR. Ubiquitin-recognition protein Ufd1 couples the endoplasmic reticulum (ER) stress response to cell cycle control. Proceedings of the National Academy of Sciences. 2011; 108: 9119-9124.

30. Faronato M, et al., The deubiquitylase USP15 stabilizes newly synthesized REST and rescues its expression at mitotic exit. Cell Cycle. 2013; 12:1964.

31. McFarlane C, et al., The deubiquitinating enzyme USP17 is highly expressed in tumor biopsies, is cell cycle regulated, and is required for G1-S progression. Cancer research. 2010; 70:3329-3339.

32. Huang X, et al., Deubiquitinase USP37 Is Activated by CDK2 to Antagonize APC CDH1 and Promote S Phase Entry. Molecular cell. 2011; 42:511-523.

33. van Leuken RJ, et al., Usp39 is essential for mitotic spindle checkpoint integrity and controls mRNA-levels of aurora B. Cell Cycle. 2008; 7:2710-2719.

34. Stegmeier F, et al., Anaphase initiation is regulated by antagonistic ubiquitination and deubiquitination activities. Nature. 2007; 446:876-881.
35. Aressy B, et al., A screen for deubiquitinating enzymes involved in the $\mathrm{g} 2 / \mathrm{m}$ checkpoint identifies usp50 as a regulator of hsp90-dependent wee1 stability. Cell Cycle. 2010; 9:3839-3846.

36. Pereg Y, et al., Ubiquitin hydrolase Dub3 promotes oncogenic transformation by stabilizing $\mathrm{Cdc} 25 \mathrm{~A}$. nature cell biology. 2010; 12:400-406.

37. Shan J, Zhao W, Gu W. Suppression of cancer cell growth by promoting cyclin D1 degradation. Molecular cell. 2009; 36:469-476.

38. Lu Y, et al., Identification of distinctive patterns of USP19mediated growth regulation in normal and malignant cells. PloS one. 2011; 6:e15936.

39. van der Horst A, et al., FOXO4 transcriptional activity is regulated by monoubiquitination and USP7/HAUSP. Nature cell biology. 2006; 8:1064-1073.

40. Song MS, et al., The deubiquitinylation and localization of PTEN are regulated by a HAUSP-PML network. Nature. 2008; 455:813-817.

41. Yoshida A, et al., CSN5/Jab1 controls multiple events in the mammalian cell cycle. FEBS letters. 2010; 584:4545-4552.

42. $\mathrm{Yu} \mathrm{H}$, et al., The ubiquitin carboxyl hydrolase BAP1 forms a ternary complex with YY1 and HCF-1 and is a critical regulator of gene expression. Molecular and cellular biology. 2010; 30:5071-5085.

43. Eletr ZM, Wilkinson KD. An emerging model for BAP1's role in regulating cell cycle progression. Cell biochemistry and biophysics. 2011; 60:3-11.

44. Nijman S, et al., The deubiquitinating enzyme USP1 regulates the Fanconi anemia pathway. Molecular cell. 2005; 17:331-339.

45. Guervilly JH, et al., USP1 deubiquitinase maintains phosphorylated CHK1 by limiting its DDB1-dependent degradation. Human molecular genetics. 2011; 20:2171-2181.

46. Huang TT, et al., Regulation of monoubiquitinated PCNA by DUB autocleavage. Nature cell biology. 2006; 8:341-347.

47. Murai J, et al., The USP1/UAF1 complex promotes double-strand break repair through homologous recombination. Molecular and cellular biology. 2011; 31:2462-2469.

48. Nishi R, et al., Systematic characterization of deubiquitylating enzymes for roles in maintaining genome integrity. Nature cell biology. 2014.

49. Al-Hakim A, et al., The ubiquitous role of ubiquitin in the DNA damage response. DNA repair. 2010; 9: 1229-1240.

50. Potu H, et al., Usp5 links suppression of p53 and FAS levels in melanoma to the BRAF pathway. Oncotarget. 2014; 5:5559.

51. Schoenfeld AR, et al., BRCA2 is ubiquitinated in vivo and interacts with USP11, a deubiquitinating enzyme that exhibits prosurvival function in the cellular response 
to DNA damage. Molecular and cellular biology. 2004; 24:7444-7455.

52. Yuan J, et al., HERC2-USP20 axis regulates DNA damage checkpoint through Claspin. Nucleic acids research. 2014; :gku1034.

53. Yuan J, et al., HERC2-USP20 axis regulates DNA damage checkpoint through Claspin. Nucleic acids research. 2014; 42:13110-13121.

54. Zhang D, et al., A role for the deubiquitinating enzyme USP28 in control of the DNA-damage response. Cell. 2006; 126:529-542.

55. Sy SM, et al., The ubiquitin specific protease USP34 promotes ubiquitin signaling at DNA double-strand breaks. Nucleic acids research. 2013; 41:8572-8580.

56. Parsons JL, et al., USP47 is a deubiquitylating enzyme that regulates base excision repair by controlling steadystate levels of DNA polymerase $\beta$. Molecular cell. 2011; 41:609-615.

57. Joo HY, et al., Regulation of cell cycle progression and gene expression by H2A deubiquitination. Nature. 2007; 449:1068-1072.

58. Clague MJ, Coulson JM, Urbé S. Deciphering histone 2A deubiquitination. Genome Biol. 2008; 9:202.

59. Atanassov BS, Koutelou E, Dent SY. The role of deubiquitinating enzymes in chromatin regulation. FEBS letters. 2011; 585:2016-2023.

60. Yu H, et al., Tumor suppressor and deubiquitinase BAP1 promotes DNA double-strand break repair. Proceedings of the National Academy of Sciences. 2014; 111:285-290.

61. Zhang Y. Transcriptional regulation by histone ubiquitination and deubiquitination. Genes \& development. 2003; 17:2733-2740.

62. Zhu P, et al., A histone H2A deubiquitinase complex coordinating histone acetylation and $\mathrm{H} 1$ dissociation in transcriptional regulation. Molecular cell. 2007; 27:609-621.

63. Zhang XY, et al., The putative cancer stem cell marker USP22 is a subunit of the human SAGA complex required for activated transcription and cell-cycle progression. Molecular cell. 2008; 29:102-111.

64. Feng L, Wang J, Chen J. The Lys63-specific deubiquitinating enzyme BRCC36 is regulated by two scaffold proteins localizing in different subcellular compartments. Journal of Biological Chemistry. 2010; 285: 30982-30988.

65. Maertens GN, et al., Ubiquitin-specific proteases 7 and 11 modulate Polycomb regulation of the INK4a tumour suppressor. The EMBO journal. 2010; 29:2553-2565.

66. Atanassov BS, et al., Gen5 and SAGA regulate shelterin protein turnover and telomere maintenance. Molecular cell. 2009; 35:352-364.

67. Yao T, et al., Distinct modes of regulation of the Uch37 deubiquitinating enzyme in the proteasome and in the Ino80 chromatin-remodeling complex. Molecular cell. 2008; 31:909-917.
68. Vogelstein B, Kinzler KW. Cancer genes and the pathways they control. Nature medicine. 2004; 10:789-799.

69. Forbes SA, et al., COSMIC: mining complete cancer genomes in the Catalogue of Somatic Mutations in Cancer. Nucleic acids research. 2010; gkq929.

70. Harris SL, Levine AJ. The p53 pathway: positive and negative feedback loops. Oncogene. 2005; 24: 2899-2908.

71. Brooks C, et al., The p53-Mdm2-HAUSP complex is involved in p53 stabilization by HAUSP. Oncogene. 2007; 26:7262-7266.

72. Kon $\mathrm{N}$, et al., Inactivation of HAUSP in vivo modulates $\mathrm{p} 53$ function. Oncogene. 2009; 29:1270-1279.

73. Stevenson LF, et al., The deubiquitinating enzyme USP2a regulates the p53 pathway by targeting Mdm2. The EMBO journal. 2007; 26:976-986.

74. Yuan J, et al., USP10 regulates p53 localization and stability by deubiquitinating p53. cell. 2010; 140:384-396.

75. Sun XX, Dai MS. Deubiquitinating enzyme regulation of the p53 pathway: A lesson from Otub1. 2014.

76. Dayal S, et al., Suppression of the deubiquitinating enzyme USP5 causes the accumulation of unanchored polyubiquitin and the activation of p53. Journal of Biological Chemistry. 2009; 284:5030-5041.

77. Liu J, et al., JTV1 co-activates FBP to induce USP29 transcription and stabilize p53 in response to oxidative stress. The EMBO journal. 2011; 30:846-858.

78. Zhang $\mathrm{X}$, et al., USP4 inhibits p53 through deubiquitinating and stabilizing ARF-BP1. The EMBO journal. 2011; 30:2177-2189.

79. Prasad S, Ravindran J, Aggarwal BB. NF- $\kappa$ B and cancer: how intimate is this relationship. Molecular and cellular biochemistry. 2010; 336:25-37.

80. Harhaj EW, Dixit VM. Deubiquitinases in the regulation of NF- $\kappa \mathrm{B}$ signaling. Cell research. 2010; 21:22-39.

81. Reiley WW, et al., Deubiquitinating enzyme CYLD negatively regulates the ubiquitin-dependent kinase Tak1 and prevents abnormal $\mathrm{T}$ cell responses. The Journal of experimental medicine. 2007; 204:1475-1485.

82. Massoumi R, et al., Cyld inhibits tumor cell proliferation by blocking Bcl-3-dependent NF- $\mathrm{kB}$ signaling. Cell. 2006; 125:665-677.

83. Reiley W, Zhang M, Sun SC. Negative regulation of JNK signaling by the tumor suppressor CYLD. Journal of Biological Chemistry. 2004; 279:55161-55167.

84. Li L, et al., The zinc finger protein A20 targets TRAF2 to the lysosomes for degradation. Biochimica et Biophysica Acta (BBA)-Molecular Cell Research. 2009; 1793:346-353.

85. Wertz IE, et al., De-ubiquitination and ubiquitin ligase domains of A20 downregulate NF- $\kappa$ B signalling. Nature. 2004; 430:694-699.

86. Takiuchi T, et al., Suppression of LUBAC-mediated linear ubiquitination by a specific interaction between LUBAC 
and the deubiquitinases CYLD and OTULIN. Genes to Cells. 2014; 19:254-272.

87. Liang $\mathrm{J}$, et al., MCP-induced protein 1 deubiquitinates TRAF proteins and negatively regulates JNK and NF- $\mathrm{KB}$ signaling. The Journal of experimental medicine. 2010; 207:2959-2973.

88. Metzig M, et al., An RNAi screen identifies USP2 as a factor required for TNF- $\alpha$-induced NF- $\kappa \mathrm{B}$ signaling. International Journal of Cancer. 2011; 129:607-618.

89. Fan Y, et al., USP4 targets TAK1 to downregulate TNF $\alpha$ induced NF- $\kappa$ B activation. Cell Death \& Differentiation. 2011; 18:1547-1560.

90. González-Navajas JM, et al., Interleukin 1 receptor signaling regulates DUBA expression and facilitates Toll-like receptor 9-driven antiinflammatory cytokine production. The Journal of experimental medicine. 2010; 207:2799-2807.

91. Xu G, et al., Ubiquitin-specific peptidase 21 inhibits tumor necrosis factor $\alpha$-induced nuclear factor $\kappa \mathrm{B}$ activation via binding to and deubiquitinating receptor-interacting protein 1. Journal of Biological Chemistry. 2010; 285:969-978.

92. Enesa K, et al., NF-кB Suppression by the Deubiquitinating Enzyme Cezanne A NOVEL NEGATIVE FEEDBACK LOOP IN PRO-INFLAMMATORY SIGNALING. Journal of Biological Chemistry. 2008; 283:7036-7045.

93. McNally RS, et al., DJ-1 enhances cell survival through the binding of Cezanne, a negative regulator of NF-kB. Journal of Biological Chemistry. 2011; 286: 4098-4106.

94. Poalas $\mathrm{K}$, et al., Negative regulation of $\mathrm{NF}-\kappa \mathrm{B}$ signaling in $\mathrm{T}$ lymphocytes by the ubiquitin-specific protease USP34. Cell Communication and Signaling. $2013 ; 11: 25$.

95. Mosesson Y, Mills GB, Yarden Y. Derailed endocytosis: an emerging feature of cancer. Nature Reviews Cancer. 2008; 8:835-850.

96. Niendorf S, et al., Essential role of ubiquitin-specific protease 8 for receptor tyrosine kinase stability and endocytic trafficking in vivo. Molecular and cellular biology. 2007; 27:5029-5039.

97. MacDonald E, Urbé S, Clague MJ. USP8 controls the trafficking and sorting of lysosomal enzymes. Traffic. 2014.

98. McCullough J, Clague MJ, Urbé S. AMSH is an endosome-associated ubiquitin isopeptidase. The Journal of cell biology. 2004; 166:487-492.

99. Clague MJ, Urbé S. Endocytosis: the DUB version. Trends in cell biology. 2006; 16:551-559.

100. Duex JE, Sorkin A. RNA interference screen identifies Usp18 as a regulator of epidermal growth factor receptor synthesis. Molecular biology of the cell. 2009; 20:1833-1844.
101. Duex JE, et al., Usp18 regulates epidermal growth factor (EGF) receptor expression and cancer cell survival via microRNA-7. Journal of Biological Chemistry. 2011; 286:25377-25386.

102. Liu H, et al., Regulation of ErbB2 receptor status by the proteasomal DUB POH1. PloS one. 2009; 4:e5544.

103. Klaus A, Birchmeier W. Wnt signalling and its impact on development and cancer. Nature Reviews Cancer. 2008; 8:387-398.

104. Tauriello DV, et al., Loss of the tumor suppressor CYLD enhances Wnt/ $\beta$-catenin signaling through K63-linked ubiquitination of Dvl. Molecular cell. 2010; 37:607-619.

105. Zhao B, et al., The ubiquitin specific protease 4 (USP4) is a new player in the Wnt signalling pathway. Journal of cellular and molecular medicine. 2009; 13:1886-1895.

106. Huang X, et al., The COP9 Signalosome Mediates $\beta$-Catenin Degradation by Deneddylation and Blocks Adenomatous Polyposis coli Destruction via USP15. Journal of molecular biology. 2009; 391: 691-702.

107. Ma P, et al., The Ubiquitin Ligase RNF220 Enhances Canonical Wnt Signaling through USP7-Mediated Deubiquitination of $\beta$-Catenin. Molecular and cellular biology. 2014; 34:4355-4366.

108. Jung H, et al., Deubiquitination of Dishevelled by Usp14 is required for Wnt signaling. Oncogenesis. 2013; 2:e64.

109. Lui TT, et al., The Ubiquitin specific protease USP34 regulates Axin stability and Wnt/ $\beta$-catenin signaling. Molecular and cellular biology. 2011.

110. Tran H, et al., Trabid, a new positive regulator of Wnt-induced transcription with preference for binding and cleaving K63-linked ubiquitin chains. Genes \& development. 2008; 22:528-542.

111. Pardali K, Moustakas A. Actions of TGF- $\beta$ as tumor suppressor and pro-metastatic factor in human cancer. Biochimica et Biophysica Acta (BBA)-Reviews on Cancer. 2007; 1775:21-62.

112. Dupont S, et al., FAM/USP9x, a deubiquitinating enzyme essential for TGF $\beta$ signaling, controls Smad4 monoubiquitination. Cell. 2009; 136:123-135.

113. Al-Hakim A, et al., Control of AMPK-related kinases by USP9X and atypical Lys29/Lys33-linked polyubiquitin chains. Biochem. J. 2008; 411:249-260.

114. Wicks SJ, et al., The deubiquitinating enzyme UCH37 interacts with Smads and regulates TGF- $\beta$ signalling. Oncogene. 2005; 24:8080-8084.

115. Yeh HM, et al., Ubiquitin-specific protease 13 regulates IFN signaling by stabilizing STAT1. The Journal of Immunology. 2013; 191:3328-3336.

116. Zhang L, et al., Ubiquitin-Specific Protease $2 b$ Negatively Regulates IFN- $\beta$ Production and Antiviral Activity by Targeting TANK-Binding Kinase 1. The Journal of Immunology. 2014; 193:2230-2237. 
117. McClurg UL, et al., Deubiquitinating enzyme Usp12 regulates the interaction between the androgen receptor and the Akt pathway. Oncotarget. 2014; 5:7081.

118. Schröder C, et al., Prognostic relevance of ubiquitin C-terminal hydrolase L1 (UCH-L1) mRNA and protein expression in breast cancer patients. Journal of cancer research and clinical oncology. 2013; 139:1745-1755.

119. Wang SS, et al., Bapl is essential for kidney function and cooperates with $\mathrm{Vhl}$ in renal tumorigenesis. Proceedings of the National Academy of Sciences. 2014; 111:16538-16543.

120. Wang X, et al., The 19S Deubiquitinase Inhibitor b-AP15 Is Enriched in Cells and Elicits Rapid Commitment to Cell Death. Molecular pharmacology. 2014; 85: 932-945.

121. Ke H, et al., Cyld inhibits melanoma growth and progression through suppression of the JNK/AP-1 and $\beta 1$-integrin signaling pathways. Journal of Investigative Dermatology. 2012; 133:221-229.

122. Pannem RR, et al., CYLD controls c-MYC expression through the JNK-dependent signaling pathway in hepatocellular carcinoma. Carcinogenesis. 2013; bgt335.

123. Kim JM, et al., Inactivation of murine Usp1 results in genomic instability and a Fanconi anemia phenotype. Developmental cell. 2009; 16:314-320.

124. Williams SA, et al., USP1 deubiquitinates ID proteins to preserve a mesenchymal stem cell program in osteosarcoma. Cell. 2011; 146:918-930.

125. Benassi B, et al., MYC is activated by USP2a-mediated modulation of microRNAs in prostate cancer. Cancer discovery. 2012; 2:236-247.

126. He X, et al., USP2a negatively regulates IL-1 $\beta$-and virusinduced NF- $\kappa \mathrm{B}$ activation by deubiquitinating TRAF6. Journal of molecular cell biology. 2013; 5:39-47.

127. Tao BB, et al., Up-regulation of USP2a and FASN in gliomas correlates strongly with glioma grade. Journal of Clinical Neuroscience. 2013; 20:717-720.

128. Nicassio F, et al., Human USP3 is a chromatin modifier required for $\mathrm{S}$ phase progression and genome stability. Current biology. 2007; 17:1972-1977.

129. Raychaudhuri P. USP3 controls BRCA1 "foci". Cell Cycle. 2014; 13:183.

130. Sharma N, et al., USP3 counteracts RNF168 via deubiquitinating $\mathrm{H} 2 \mathrm{~A}$ and gammaH2AX at lysine 13 and 15 . Cell Cycle. 2014; 13:106-114.

131. Hock AK, et al., Regulation of p53 stability and function by the deubiquitinating enzyme USP42. The EMBO journal. 2011; 30:4921-4930.

132. Zhang L, et al., USP4 is regulated by AKT phosphorylation and directly deubiquitylates TGF-[beta] type I receptor. Nature cell biology. 2012; 14:717-726.
133. Izaguirre DI, et al., PTBP1-dependent regulation of USP5 alternative RNA splicing plays a role in glioblastoma tumorigenesis. Molecular carcinogenesis. 2012; 51:895-906.

134. Nakajima S, et al., Ubiquitin-Specific Protease 5 Is Required for the Efficient Repair of DNA Double-Strand Breaks. PloS one. 2014; 9:e84899.

135. Oliveira AM, et al., USP6 (Tre2) fusion oncogenes in aneurysmal bone cyst. Cancer Research. 2004; 64:1920-1923.

136. Pringle LM, et al., Atypical mechanism of NF- $\mathrm{BB}$ activation by TRE17/ubiquitin-specific protease 6 (USP6) oncogene and its requirement in tumorigenesis. Oncogene. 2011; 31:3525-3535.

137. Ye Y, et al., TRE17/USP6 oncogene translocated in aneurysmal bone cyst induces matrix metalloproteinase production via activation of NF- $\mathrm{\kappa B}$. Oncogene. 2010; 29:3619-3629.

138. Chauhan D, et al., A small molecule inhibitor of ubiquitin-specific protease-7 induces apoptosis in multiple myeloma cells and overcomes bortezomib resistance. Cancer cell. 2012; 22:345-358.

139. Cheon KW, Baek KH. HAUSP as a therapeutic target for hematopoietic tumors (review). International journal of oncology. 2006; 28:1209-1215.

140. Berlin I, et al., The deubiquitinating enzyme USP8 promotes trafficking and degradation of the chemokine receptor 4 at the sorting endosome. Journal of Biological Chemistry. 2010; 285:37895-37908.

141. Byun S, et al., USP8 is a novel target for overcoming gefitinib resistance in lung cancer. Clinical Cancer Research. 2013; 19:3894-3904.

142. Panner A, et al., Ubiquitin-specific protease 8 links the PTEN-Akt-AIP4 pathway to the control of FLIPS stability and TRAIL sensitivity in glioblastoma multiforme. Cancer research. 2010; 70:5046-5053.

143. Xia R, et al., USP8 promotes smoothened signaling by preventing its ubiquitination and changing its subcellular localization. PLoS biology. 2012; 10:e1001238.

144. Schwickart M, et al., Deubiquitinase USP9X stabilizes MCL1 and promotes tumour cell survival. Nature. 2009; 463:103-107.

145. Wang S, et al., Ablation of the oncogenic transcription factor ERG by deubiquitinase inhibition in prostate cancer. Proceedings of the National Academy of Sciences. 2014; 111:4251-4256.

146. Cox JL, et al., The SOX2-Interactome in Brain Cancer Cells Identifies the Requirement of MSI2 and USP9X for the Growth of Brain Tumor Cells. PloS one. 2013; 8:e62857. 
147. Peng J, et al., USP9X expression correlates with tumor progression and poor prognosis in esophageal squamous cell carcinoma. Diagnostic pathology. 2013; 8:177.

148. Sun $\mathrm{H}$, et al., Bcr-Abl ubiquitination and Usp9x inhibition block kinase signaling and promote CML cell apoptosis. Blood. 2011; 117:3151-3162.

149. Sun C, et al., An azoospermic man with a de novo point mutation in the Y-chromosomal gene USP9Y. Nature genetics. 1999; 23:429-432.

150. Deng S, et al., Over-expression of genes and proteins of ubiquitin specific peptidases (USPs) and proteasome subunits (PSs) in breast cancer tissue observed by the methods of RFDD-PCR and proteomics. Breast cancer research and treatment. 2007; 104:21-30.

151. Lin Z, et al., USP10 Antagonizes c-Myc Transcriptional Activation through SIRT6 Stabilization to Suppress Tumor Formation. Cell reports. 2013; 5:1639-1649.

152. Zeng Z, et al., Prognostic significance of USP10 as a tumor-associated marker in gastric carcinoma. Tumor Biology. 2014; 35:3845-3853.

153. Al-Salihi MA, et al., USP11 augments TGF $\beta$ signalling by deubiquitylating ALK5. Open biology. 2012; 2:120063.

154. Bayraktar S, et al., USP-11 as a predictive and prognostic factor following neoadjuvant therapy in women with breast cancer. Cancer journal (Sudbury, Mass.). 2013; 19:10

155. Burkhart RA, et al., Mitoxantrone targets human ubiquitin-specific peptidase 11 (USP11) and is a potent inhibitor of pancreatic cancer cell survival. Molecular Cancer Research. 2013; 11:901-911.

156. de La Vega M, et al., The deubiquitinating enzyme USP17 is essential for GTPase subcellular localization and cell motility. Nature communications. 2011; 2:259.

157. Tian Z, et al., A novel small molecule inhibitor of deubiquitylating enzyme USP14 and UCHL5 induces apoptosis in multiple myeloma and overcomes bortezomib resistance. Blood. 2014; 123:706-716.

158. Shinji S, et al., Ubiquitin-specific protease 14 expression in colorectal cancer is associated with liver and lymph node metastases. Oncology reports. 2006; 15:539-543.

159. $\mathrm{Wu} \mathrm{N}$, et al., Over-Expression of Deubiquitinating Enzyme USP14 in Lung Adenocarcinoma Promotes Proliferation through the Accumulation of $\beta$-Catenin. International journal of molecular sciences. 2013; 14:10749-10760.

160. Eichhorn PJ, et al., USP15 stabilizes TGF-[beta] receptor I and promotes oncogenesis through the activation of TGF-[beta] signaling in glioblastoma. Nature medicine. 2012; 18:429-435.

161. Inui M, et al., USP15 is a deubiquitylating enzyme for receptor-activated SMADs. Nature cell biology. 2011; 13:1368-1375.
162. Gelsi-Boyer V, et al., Genome profiling of chronic myelomonocytic leukemia: frequent alterations of RAS and RUNX1 genes. BMC cancer. 2008; 8:299.

163. Ernst A, et al., A strategy for modulation of enzymes in the ubiquitin system. Science. 2013; 339:590-595.

164. Ye Y, et al., Polyubiquitin binding and cross-reactivity in the USP domain deubiquitinase USP21. EMBO reports. 2011; 12:350-357.

165. McFarlane $\mathrm{C}$, et al., The deubiquitinating enzyme USP17 is associated with nonsmall cell lung cancer (NSCLC) recurrence and metastasis. Oncotarget. 2013; 4:1836.

166. Liu LQ, et al., A novel ubiquitin-specific protease, UBP43, cloned from leukemia fusion protein AML1ETO-expressing mice, functions in hematopoietic cell differentiation. Molecular and cellular biology. 1999; 19:3029-3038.

167. Yan M, et al., Ubp43 regulates BCR-ABL leukemogenesis via the type 1 interferon receptor signaling. Blood. 2007; 110:305-312.

168. Hassink GC, et al., The ER-resident ubiquitin-specific protease 19 participates in the UPR and rescues ERAD substrates. EMBO reports. 2009; 10:755-761.

169. Lee JG, et al., Characterization of the deubiquitinating activity of USP19 and its role in endoplasmic reticulumassociated degradation. Journal of Biological Chemistry. 2014; 289:3510-3517.

170. Li Z, et al., Identification of a deubiquitinating enzyme subfamily as substrates of the von Hippel-Lindau tumor suppressor. Biochemical and biophysical research communications. 2002; 294:700-709.

171. Yasunaga J, et al., Ubiquitin-specific peptidase 20 targets TRAF6 and human T cell leukemia virus type 1 tax to negatively regulate NF- $\kappa \mathrm{B}$ signaling. Journal of virology. 2011; 85:6212-6219.

172. Riester M, et al., Integrative Analysis of 1q23. 3 CopyNumber Gain in Metastatic Urothelial Carcinoma. Clinical Cancer Research. 2014; 20:1873-1883.

173. Dai W, et al., Ubiquitin-Specific Peptidase 22, a Histone Deubiquitinating Enzyme, Is a Novel Poor Prognostic Factor for Salivary Adenoid Cystic Carcinoma. PloS one. 2014; 9:e87148.

174. Liang J, et al., Ubiquitin-specific protease 22: a novel molecular biomarker in glioma prognosis and therapeutics. Medical Oncology. 2014; 31:1-6.

175. Ning J, et al., Overexpression of ubiquitin-specific protease 22 predicts poor survival in patients with earlystage non-small cell lung cancer. European journal of histochemistry: EJH. 2012; 56.

176. Piao S, et al., Increased expression of USP22 is associated with disease progression and patient prognosis of salivary duct carcinoma. Oral oncology. 2013; 49:796-801. 
177. Schrecengost RS, et al., USP22 Regulates Oncogenic Signaling Pathways to Drive Lethal Cancer Progression. Cancer research. 2014; 74:272-286.

178. Wang H, et al., Prognostic significance of USP22 as an oncogene in papillary thyroid carcinoma. Tumor Biology. 2013; 34:1635-1639.

179. Blount JR, et al., Ubiquitin-specific protease 25 functions in endoplasmic reticulum-associated degradation. PloS one. 2012; 7:e36542.

180. Zhong B, et al., Ubiquitin-specific protease 25 regulates TLR4-dependent innate immune responses through deubiquitination of the adaptor protein TRAF3. Science signaling. 2013; 6:ra35.

181. A Paduch D, Mielnik A, Schlegel PN. Novel mutations in testis-specific ubiquitin protease 26 gene may cause male infertility and hypogonadism. Reproductive biomedicine online. 2005; 10:747-754.

182. Stouffs K, et al., Possible role of USP26 in patients with severely impaired spermatogenesis. European journal of human genetics. 2004; 13:336-340.

183. Wang PJ, et al., An abundance of X-linked genes expressed in spermatogonia. Nature genetics. 2001; 27:422-426.

184. Martín Y, et al., USP29 controls the stability of checkpoint adaptor Claspin by deubiquitination. Oncogene. 2014.

185. Akhavantabasi S, et al., USP32 is an active, membranebound ubiquitin protease overexpressed in breast cancers. Mammalian Genome. 2010; 21:388-397.

186. Schulte I, et al., Structural analysis of the genome of breast cancer cell line ZR-75-30 identifies twelve expressed fusion genes. BMC genomics. 2012; 13:719.

187. Li Z, et al., Ubiquitination of a novel deubiquitinating enzyme requires direct binding to von Hippel-Lindau tumor suppressor protein. Journal of Biological Chemistry. 2002; 277:4656-4662.

188. De Pittà C, et al., A leukemia-enriched cDNA microarray platform identifies new transcripts with relevance to the biology of pediatric acute lymphoblastic leukemia. haematologica. 2005; 90:890-898.

189. Paulsson K, et al., A novel and cytogenetically cryptic $\mathrm{t}(7 ; 21)(\mathrm{p} 22 ; \mathrm{q} 22)$ in acute myeloid leukemia results in fusion of RUNX1 with the ubiquitin-specific protease gene USP42. Leukemia. 2005; 20:224-229.

190. Delgado-Díaz MR, et al., Dub3 controls DNA damage signalling by direct deubiquitination of H2AX. Molecular oncology. 2014.

191. Zeng LX, Tang Y, Ma Y. Ataxin-3 expression correlates with the clinicopathologic features of gastric cancer. Int J Clin Exp Med. 2014; 7:973-981.

192. Sacco J, et al., The deubiquitylase Ataxin-3 restricts PTEN transcription in lung cancer cells. Oncogene. 2013.
193. Switonski PM, et al., Mouse ataxin-3 functional knock-out model. Neuromolecular medicine. 2011; 13:54-65.

194. da Silva CG, et al., A20 ExprEssing Tumors And AnTicAncEr drug rEsisTAncE. 2014

195. Skrott Z, Cvek B. Diethyldithiocarbamate complex with copper: the mechanism of action in cancer cells. Mini reviews in medicinal chemistry. 2012; 12:1184-1192.

196. Schütz AK, et al., Role of CSN5/JAB1 in Wnt/ $\beta$-catenin activation in colorectal cancer cells. FEBS letters. 2012; 586:1645-1651.

197. Pan Y, Yang H, Claret FX. Emerging roles of Jab1/CSN5 in DNA damage response, DNA repair, and cancer. Cancer biology \& therapy. 2014; 15:256-262.

198. Rebbeck TR, et al., Modification of BRCA1-associated breast and ovarian cancer risk by BRCA1-interacting genes. Cancer research. 2011; 71:5792-5805.

199. Dexheimer TS, et al., Synthesis and Structure-Activity Relationship Studies of N-Benzyl-2-phenylpyrimidin-4amine Derivatives as Potent USP1/UAF1 Deubiquitinase Inhibitors with Anticancer Activity against Nonsmall Cell Lung Cancer. Journal of medicinal chemistry. 2014; 57:8099-8110.

200. Okada K, et al., Vialinin A is a ubiquitin-specific peptidase inhibitor. Bioorganic \& medicinal chemistry letters. 2013; 23:4328-4331.

201. Kessler BM. Selective and reversible inhibitors of ubiquitin-specific protease 7: a patent evaluation (WO2013030218). Expert opinion on therapeutic patents. 2014; 24:597-602.

202. Daviet L, Colland F. Targeting ubiquitin specific proteases for drug discovery. Biochimie. 2008; 90:270-283.

203. D'Arcy P, et al., A Novel Small Molecule Inhibitor Of Deubiquitylating Enzyme USP14 and UCHL5 Induces Apoptosis In Myeloma Cells and Overcomes Bortezomib Resistance. Blood. 2013; 122:1923-1923.

204. Chen X, et al., Anti-rheumatic agent auranofin induced apoptosis in chronic myeloid leukemia cells resistant to imatinib through both Bcr/Abl-dependent and-independent mechanisms. Oncotarget. 2014; 5:p. 9118.

205. Mermerian AH, et al., Structure-activity relationship, kinetic mechanism, and selectivity for a new class of ubiquitin C-terminal hydrolase-L1 (UCH-L1) inhibitors. Bioorganic \& medicinal chemistry letters. 2007; 17:3729-3732.

206. Mullally JE, et al., Cyclopentenone prostaglandins of the $\mathrm{J}$ series inhibit the ubiquitin isopeptidase activity of the proteasome pathway. Journal of Biological Chemistry. 2001; 276:30366-30373.

207. Altun M, et al., Activity-based chemical proteomics accelerates inhibitor development for deubiquitylating enzymes. Chemistry \& biology. 2011; 18:1401-1412. 